\title{
Structure Theorem for Functionals in the Space $\mathfrak{S}_{\omega_{1}, \omega_{2}}^{\prime}$
}

\author{
Hamed M. Obiedat, Wasfi A. Shatanawi, and Mohd M. Yasein \\ Department of Mathematics, Hashemite University, P.O. Box 150459, \\ Zarqa 13115, Jordan \\ Correspondence should be addressed to Hamed M. Obiedat, hobiedat@hu.edu.jo
}

Received 19 August 2007; Revised 30 September 2007; Accepted 22 November 2007

Recommended by Manfred H. Moller

We introduce the space $\mathfrak{S}_{\omega_{1}, \omega_{2}}$ of all $C^{\infty}$ functions $\varphi$ such that $\sup _{|\alpha| \leq m}\left\|e^{k \omega_{1}} \partial^{\alpha} \varphi\right\|_{\infty}$ and $\sup _{|\alpha| \leq m}\left\|e^{k \omega_{2}} \partial^{\alpha} \hat{\varphi}\right\|_{\infty}$ are finite for all $k \in \mathbb{N}_{0}, \alpha \in \mathbb{N}_{0}^{n}$, where $\omega_{1}$ and $\omega_{2}$ are two weights satisfying the classical Beurling conditions. Moreover, we give a topological characterization of the space $\mathfrak{S}_{\omega_{1}, \omega_{2}}$ without conditions on the derivatives. For functionals in the dual space $\mathfrak{S}_{\omega_{1}, \omega_{2}}^{\prime}$, we prove a structure theorem by using the classical Riesz representation thoerem.

Copyright (C) 2008 Hamed M. Obiedat et al. This is an open access article distributed under the Creative Commons Attribution License, which permits unrestricted use, distribution, and reproduction in any medium, provided the original work is properly cited.

\section{Introduction}

The theory of ultradistributions introduced by Beurling [1] was to find an appropriate context for his work on almost holomorphic extensions. Beurling proved that ultradistributions are limits of holomorphic functions in the upper and lower half-planes. Björck [2] studied and expanded the theory of Beurling on ultradistributions to extend the work of Hörmander [3] on existence, nonexistence, and regularity of solutions of constant coefficient linear partial differential equations.

The Beurling-Björck space $\mathfrak{S}_{w}$, as defined in [2], consists of $C^{\infty}$ functions such that the functions and their Fourier transform jointly with all their derivatives decay ultrarapidly at infinity.

In this paper, we introduce the space $\mathfrak{S}_{w_{1}, w_{2}}$ of $C^{\infty}$ functions such that the functions and their Fourier transform jointly with all their derivatives decay ultrarapidly at infinity. Moreover, we give a characterization of the space $\mathfrak{S}_{w_{1}, w_{2}}$ and its dual $\mathfrak{S}_{w_{1}, w_{2}}^{\prime}$.

The main difference between the Beurling-Björck space $\mathfrak{S}_{w}$ and the space $\mathfrak{S}_{w_{1}, w_{2}}$ is that the decay of the functions in $\mathfrak{S}_{w}$ and their Fourier transform are measured by the same submultiplicative function $e^{k w}, k \geq 0$. Whereas the decay of the functions in $\mathfrak{S}_{w_{1}, w_{2}}$ and 
their Fourier transform are measured by two different submultiplicative functions $e^{k w_{1}}$ and $e^{k w_{2}}, k \geq 0$.

This paper is organized in three sections. In Section 2, we give preliminary definitions and results and introduce the space $\mathfrak{S}_{w_{1}, w_{2}}$. In Section 3, we give a topological characterization of the space $\mathfrak{S}_{w_{1}, w_{2}}$ without conditions on the derivatives. In Section 4 , we use the topological characterization of the space $\mathfrak{S}_{w_{1}, w_{2}}$ that is given in Section 3 to prove a representation theorem for functionals in the dual space $\mathfrak{S}_{w_{1}, w_{2}}^{\prime}$ of the space $\mathfrak{S}_{w_{1}, w_{2}}$.

The symbols $C^{\infty}, C_{0}^{\infty}, L^{p}$, and so forth indicate the usual spaces of functions defined on $\mathbb{R}^{n}$, with complex values. We denote by $|\cdot|$ the Euclidean norm on $\mathbb{R}^{n}$, while $\|\cdot\|_{\infty}$ indicates the norm in the space $L^{\infty}$. When we do not work on the general Euclidean space $\mathbb{R}^{n}$, we will write $L^{p}(\mathbb{R})$, and so forth as appropriate. Partial derivatives will be denotedby $\partial^{\alpha}$, where $\alpha$ is a multiindex $\left(\alpha_{1}, \ldots, \alpha_{n}\right)$. If it is necessary to indicate on which variables we are taking the derivative, we will do so by attaching subindexes. We will use the standard abbreviations $|\alpha|=\alpha_{1}+\cdots+\alpha_{n}$, $x^{\alpha}=x_{1}^{\alpha_{1}}, \ldots, x_{n}^{\alpha_{n}}$. With $\alpha \leq \beta$, we mean that $\alpha_{j} \leq \beta_{j}$ for every $j$. The Fourier transform of a function $g$ will be denoted by $\mathcal{F}(g)$ or $\hat{g}$ and it will be defined as $\int_{\mathbb{R}^{n}} e^{-2 \pi i x \xi} g(x) d x$. The inverse Fourier transform is then $\mathcal{F}^{-1}(g)=\int_{\mathbb{R}^{n}} e^{2 \pi i x \xi} g(\xi) d \xi$. The letter $C$ will indicate a positive constant, that may be different at different occurrences. If it is important to indicate that a constant depends on certain parameters, we will do so by attaching subindexes to the constant. We will not indicate the dependence of constants on the dimension $n$ or other fixed parameters.

\section{Preliminary definitions and results}

In this section, we give definitions and results which we will use later.

Definition 2.1 (see [2]). With $\boldsymbol{M}_{c}$, we denote the space of functions $w: \mathbb{R}^{n} \rightarrow \mathbb{R}$ of the form $w(x)=\Omega(|x|)$, where

(1) $\Omega:[0, \infty) \rightarrow[0, \infty)$ is increasing, continuous, and concave,

(2) $\Omega(0)=0$,

(3) $\int_{\mathbb{R}} \Omega\left((t) /\left(1+t^{2}\right)\right) d t<\infty$,

(4) $\Omega(t) \geq a+b \ln (1+t)$ for some $a \in \mathbb{R}$ and some $b>0$.

Standard classes of functions $w$ in $\boldsymbol{M}_{c}$ are given by

$$
w(x)=|x|^{d} \text { for } 0<d<1, \quad w(x)=p \ln (1+|x|) \quad \text { for } p>0 .
$$

Remark 2.2. Let us observe for future use that if we take an integer $N>(n / b)$, then

$$
C_{N}=\int_{\mathbb{R}^{n}} e^{-N w(x)} d x<\infty, \quad \forall w \in \mathcal{M}_{c}
$$

where $b$ is the constant in condition 4 of Definition 2.1.

The following lemma was observed in [2] without proof. Our proof is an adaptation of [4, Proposition 4.6].

Lemma 2.3. Conditions 1 and 2 in Definition 2.1 imply that $w$ is subadditive for all $w \in \boldsymbol{M}_{c}$. 
Proof. Let $0<k<1$. Since $\Omega$ is increasing, we obtain

$$
\begin{aligned}
w(x+y) & \leq \Omega\left(\frac{k}{k}|x|+\frac{1-k}{1-k}|y|\right) \\
& \leq \max \left\{\Omega\left(\frac{|x|}{k}\right), \Omega\left(\frac{|y|}{1-k}\right)\right\}
\end{aligned}
$$

Since $\Omega$ is concave on $[0, \infty)$ and $\Omega(0)=0$, we have

$$
\Omega\left(\frac{k}{k}|x|\right) \geq k \Omega\left(\frac{|x|}{k}\right), \quad \Omega\left(\frac{|y|}{1-k}\right) \geq \frac{1}{1-k} \Omega(|y|) .
$$

If we take

$$
k=\frac{\Omega(|x|)}{\Omega(|x|)+\Omega(|y|)}
$$

then we have

$$
\begin{aligned}
w(x+y) & \leq \max \left\{\Omega\left(\frac{|x|}{k}\right), \Omega\left(\frac{|y|}{1-k}\right)\right\} \\
& \leq w(x)+w(y) .
\end{aligned}
$$

This completes the proof of Lemma 2.3.

We now recall a topological characterization of the Beurling-Björck space $\mathfrak{S}_{w}$ of test functions for tempered ultradistributions.

Theorem 2.4 (see [5]). Given $w \in \mathcal{M}_{c}$, the space $\mathfrak{S}_{w}$ can be described both as a set and as a topology by

$$
\mathfrak{S}_{w}=\left\{\varphi: \mathbb{R}^{n} \mathbb{C}: \varphi \text { is continuous and for all } k=0,1,2, \ldots, p_{k, 0}(\varphi)<\infty, p_{k, 0} \circ \mathcal{F}(\varphi)<\infty\right\},
$$

where $p_{k, 0}(\varphi)=\left\|e^{k w} \varphi\right\|_{\infty}$ and $p_{k, 0} \circ \mathcal{F}(\varphi)=\left\|e^{k w} \widehat{\varphi}\right\|_{\infty}$.

We observe that $\mathfrak{S}_{w}$ becomes the Schwartz space $\mathfrak{S}$ when

$$
w(x)=\ln (1+|x|)
$$

For $\alpha, \beta>0$, the Gelfand-Shilov space $S_{\alpha}^{\beta}$ of type $S$ is characterized in [6] by the space of all $C^{\infty}$ functions $\varphi: \mathbb{R}^{n} \rightarrow \mathbb{C}$ for which the seminorms

$$
\left\|e^{k|x|^{1 / \alpha}} \varphi\right\|_{\infty}, \quad\left\|e^{m|x|^{1 / \beta}} \hat{\varphi}\right\|_{\infty}
$$

are finite for some $k, m \in \mathbb{N}_{0}$. 
Definition 2.5. Given $w_{1}, w_{2} \in \mathcal{M}_{c}$, the space $\mathfrak{S}_{w_{1}, w_{2}}$ is the space of all $C^{\infty}$ functions $\varphi: \mathbb{R}^{n} \rightarrow \mathbb{C}$ for which the seminorms

$$
p_{k, m}(\varphi)=\sup _{|\beta| \leq m}\left\|e^{k w_{1}} \partial^{\beta} \varphi\right\|_{\infty}, \quad \pi_{k, m}(\varphi)=\sup _{|\beta| \leq m}\left\|e^{k w_{2}} \partial^{\beta} \widehat{\varphi}\right\|_{\infty}
$$

are finite, for $k, m \in \mathbb{N}_{0}$ and $\beta \in \mathbb{N}_{0}^{n}$.

We can assign to $\mathfrak{S}_{w_{1}, w_{2}}$ a structure to Fréchet space by means of the countable family of seminorms

$$
S=\left\{p_{k, m}, \pi_{k, m}\right\}_{k, m=0}^{\infty} .
$$

Since $p_{k, m}(\varphi)<\infty$ for all $k=0,1,2, \ldots, \varphi$ is integrable, so $\widehat{\varphi}$ is well defined and the formulation of the condition $\pi_{k, m}(\varphi)$ makes sense for all $k=0,1,2, \ldots$

The space $\mathfrak{S}_{w_{1}, w_{2}}$, equipped with the family of seminorms

$$
S=\left\{p_{k, m}, \pi_{k, m}: k, m \in \mathbb{N}_{0}\right\},
$$

is a Fréchet space.

We observe that the space $\mathfrak{S}_{w_{1}, w_{2}}$ becomes the Beurling-Björck space $\mathfrak{S}_{w_{1}}$, when $w_{1}=w_{2}$. When $w_{2}(x)=\ln (1+|x|)$, the space of $C^{\infty}$ functions with compact support $\mathfrak{D}$ is dense subspace of $\mathfrak{S}_{w_{1}, w_{2}}$ for all $w_{1} \in \mathcal{M}_{c}$. The conditions imposed on the function $w$ assure that the space $\mathfrak{S}_{w_{1}, w_{2}}$ satisfies the properties expected from a space of testing functions. For instance, the operators of differentiation and multiplication by $x^{\alpha}$ are continuous from $\mathfrak{S}_{w_{1}, w_{2}}$ into themselves, the space $\mathfrak{S}_{w_{1}, w_{2}}$ is a topological algebra under pointwise multiplication and convolution. Unfortunately, the Fourier transformation on $\mathfrak{S}_{w_{1}, w_{2}}$ is not a topological isomorphism from $\mathfrak{S}_{w_{1}, w_{2}}$ into itself for some $w_{1}, w_{2} \in \mathcal{M}_{c}$. For Example, if we take $w_{1}(x)=|x|^{1 / 2}, w_{2}(x)=\ln (1+|x|)$, and $f \in \mathfrak{D} \backslash \mathfrak{D}_{w_{1}}$, then $f \in \mathfrak{S}_{w_{1}, w_{2}}$ but $\widehat{f} \notin \mathfrak{S}_{w 1, w 2}$; see $[1,2]$.

Theorem 2.6 (Riesz representation theorem [7]). Given a functional $L$ in the topological dual of the space $\mathcal{C}_{0}$, there exists a unique regular complex Borel measure $\mu$ such that

$$
L(\varphi)=\int_{\mathbb{R}^{n}} \varphi d \mu .
$$

Moreover, the norm of the functional $L$ is equal to the total variation $|\mu|$ of the measure $\mu$. Conversely, any such measure $\mu$ defines a continuous linear functional on $\mathcal{C}_{0}$.

We conclude this section with Lemma 2.7 [8], the version of which is due to Hadamard [9], see also [10].

Lemma 2.7 (see $[8,10]$ ). Let $f: \mathbb{R} \rightarrow \mathbb{R}$ be a continuous function with continuous derivatives of order $\leq 2$. Assume that there exist $P, Q \geq 0$ such that

$$
\begin{gathered}
|f(x)| \leq P, \\
\left|f^{\prime \prime}(x)\right| \leq Q,
\end{gathered}
$$

for all $x \in \mathbb{R}$. Then

$$
\left|f^{\prime}(x)\right| \leq \sqrt{2 P Q}
$$

for all $x \in \mathbb{R}$. 


\section{Topological characterization of the space $\mathfrak{S}_{w_{1}, w_{2}}$}

In this section, we present the following characterization of the space $\mathfrak{S}_{w_{1}, w_{2}}$, which imposes no conditions on the derivative.

Theorem 3.1. Given $w_{1}, w_{2} \in \mathcal{M}_{c}$, the space $\mathfrak{S}_{w_{1}, w_{2}}$ can be described as a set and as a topology by

$$
\mathfrak{S}_{w_{1}, w_{2}}=\left\{\varphi: \mathbb{R}^{n} \longrightarrow \mathbb{C}: \varphi \text { is continuous and for all } k=0,1,2, \ldots, p_{k, 0}(\varphi)<\infty, \pi_{k, 0}(\varphi)<\infty\right\},
$$

where $p_{k, 0}(\varphi)=\left\|e^{k w_{1}} \varphi\right\|_{\infty}, \pi_{k, 0}(\varphi)=\left\|e^{k w_{2}} \widehat{\varphi}\right\|_{\infty}$.

Proof. Let us denote by $\mathfrak{B}_{w_{1}, w_{2}}$ the space defined in (3.1). The conditions $p_{k, 0}(\varphi)$ and $\pi_{k, 0}(\varphi)$ imply the smoothness of $\varphi$ and $\hat{\varphi}$. The space $\mathfrak{B}_{w_{1}, w_{2}}$ becomes a Fréchet space with respect to the family of norms

$$
B=\left\{p_{k, 0}, \pi_{k, 0}\right\}_{k=0}^{\infty} .
$$

From these definitions, it is clear that $\mathfrak{S}_{w_{1}, w_{2}} \subseteq \mathfrak{B}_{w_{1}, w_{2}}$ and that the inclusion is continuous. To prove the converse, we use the induction on $|\beta|$ and the general idea of Landau's inequality. Fix $\varphi \in \mathfrak{B}_{w_{1}, w_{2}} \backslash\{0\}$. We want to show that $\left\|e^{k w_{1}(x)} \partial^{\beta} \varphi\right\|_{\infty}$ and $\left\|e^{k w_{2}(\xi)} \partial^{\beta} \hat{\varphi}\right\|_{\infty}$ are finite, for every $k=0,1,2, \ldots$ and every multi-index $\beta$, which is true for all $k$, when $\beta=0$. We assume that it is true for all $k$, when $|\beta| \leq m$, and we want to prove it for all $k$ and for $|\beta|=m+1$. We start with $\left\|e^{k w_{1}} \partial^{\beta} \varphi\right\|_{\infty}$. Assume that $\beta=\left(\beta_{1}+1, \beta_{2}, \ldots, \beta_{n}\right)$ with $\beta_{1}+\beta_{2}+\cdots+\beta_{n}=m, m=0,1,2, \ldots$ We also indicate $\beta^{\prime}=\left(\beta_{1}, \beta_{2}, \ldots, \beta_{n}\right), \partial^{\beta} \varphi=\partial_{x_{1}} \partial^{\beta^{\prime}} \varphi, f_{x^{\prime}}\left(x_{1}\right)=\partial^{\beta^{\prime}} \varphi\left(x_{1}, x^{\prime}\right)$ for $x^{\prime}=\left(x_{2}, \ldots, x_{n}\right)$ fixed, $\partial^{\beta} \varphi(x)=f_{x^{\prime}}^{\prime}\left(x_{1}\right)$. Moreover, if $h \neq 0$, we have

$$
f_{x^{\prime}}\left(x_{1}+h\right)=f_{x^{\prime}}\left(x_{1}\right)+f_{x^{\prime}}^{\prime}\left(x_{1}\right) h+\frac{1}{2} f_{x^{\prime}}^{\prime \prime}(y) h^{2},
$$

where $y$ is a number between $x_{1}$ and $x_{1}+h$. Thus,

$$
\left|f_{x^{\prime}}^{\prime}\left(x_{1}\right)\right| \leq \frac{\left|f_{x^{\prime}}\left(x_{1}+h\right)\right|+\left|f_{x^{\prime}}\left(x_{1}\right)\right|}{|h|}+\frac{|h|}{2}\left|f_{x^{\prime}}^{\prime \prime}(y)\right|
$$

We can write

$$
\begin{gathered}
\left|e^{k w_{1}\left(x_{1}+h, x^{\prime}\right)} f_{x^{\prime}}\left(x_{1}+h\right)\right| \leq\left|e^{k w_{1}\left(x_{1}+h, x^{\prime}\right)} \partial^{\beta^{\prime}} \varphi\left(x_{1}, x^{\prime}\right)\right| \leq q_{k, m}(\varphi), \\
\left|e^{k w_{1}(x)} f_{x^{\prime}}(x)\right| \leq q_{k, m}(\varphi) .
\end{gathered}
$$

If we take $h$ with the same sign as $x_{1}$, we have

$$
w_{1}(x) \leq w_{1}\left(x_{1}+h, x^{\prime}\right)
$$

That is,

$$
\left|f_{x^{\prime}}\left(x_{1}+h\right)\right|+\left|f_{x^{\prime}}\left(x_{1}\right)\right| \leq C_{m} p_{k, m}(\varphi) e^{-k w_{1}(x)} .
$$


To estimate $f_{x^{\prime}}^{\prime \prime}(y)=\partial_{x_{1}} \partial^{\beta} \varphi(y)$, we write

$$
\begin{aligned}
\left|\partial_{x_{1}} \partial^{\beta} \varphi(y)\right| & =\left|\partial_{x_{1}} \widehat{\widehat{\partial^{\beta}}}(y)\right| \\
& \leq \int_{\mathbb{R}^{n}}\left|2 \pi i \xi_{1}(2 \pi i \xi)^{\beta} \widehat{\varphi}(\xi)\right| d \xi \\
& \leq C_{\beta, m} \int_{\mathbb{R}^{n}}(1+|\xi|)^{m+2} e^{-r w_{2}(\xi)} e^{r w_{2}(\xi)}|\widehat{\varphi}(\xi)| d \xi,
\end{aligned}
$$

where $r>(m+n+2) / b$ is an integer and $b$ is the constant in condition 4 of Definition 2.1:

$$
\left|\partial_{x_{1}} \partial^{\beta} \varphi(y)\right| \leq C_{m} \pi_{r, 0}(\varphi) .
$$

Thus, we have

$$
\left|\partial_{x_{1}} \partial^{\beta} \varphi(y)\right| \leq C_{m} \pi_{r, 0}(\varphi),
$$

that is,

$$
\left|\partial^{\beta} \varphi(x)\right| \leq C_{m}\left[\frac{1}{t} p_{k, m}(\varphi) e^{-k w_{1}(x)}+t \pi_{r, 0}(\varphi)\right]
$$

for all $t>0$. As a function of $t$, the right side of (3.11) has a global minimum at

$$
t=\left(p_{k, m}(\varphi) e^{-k w_{1}(x)}\right)^{1 / 2}\left(\pi_{r, 0}(\varphi)\right)^{-1 / 2} .
$$

Thus, we obtain the inequality

$$
\left|\partial^{\beta} \varphi(x)\right| \leq C_{m}\left(p_{k, m}(\varphi)\right)^{1 / 2}\left(\pi_{r, 0}(\varphi)\right)^{1 / 2} e^{(-k / 2) w_{1}(x)},
$$

that is,

$$
\left|e^{k w_{1}(x)} \partial^{\beta} \varphi(x)\right| \leq C_{m}\left(p_{2 k, m}(\varphi)\right)^{1 / 2}\left(\pi_{r, 0}(\varphi)\right)^{1 / 2} .
$$

An argument, similar to the one leading to (3.14), produces

$$
\left|e^{k w_{2}(\xi)} \partial^{\beta} \widehat{\varphi}(\xi)\right| \leq C_{m}\left(\pi_{2 k, m}(\varphi)\right)^{1 / 2}\left(p_{r, 0}(\varphi)\right)^{1 / 2} .
$$

Combining (3.14), (3.15), the inductive hypothesis implies that $\varphi \in \mathfrak{S}_{w}$. The open mapping theorem can provide once again the continuity of the inclusion. However, solving the recursive inequalities (3.14), (3.15), we obtain

$$
\begin{aligned}
\left|e^{k w(x)} \partial^{\beta} \varphi(x)\right| & \leq C_{m}\left(p_{2^{m+1} k, 0}(\varphi)\right)^{2^{-m-1}}\left(\pi_{r, 0}(\varphi)\right)^{1-2^{-m-1}}, \\
\left|e^{k w(\xi)} \partial^{\beta} \widehat{\varphi}(\xi)\right| & \leq C_{m}\left(\pi_{2^{m+1} k, 0} \circ \mathcal{F}(\varphi)\right)^{2^{-m-1}}\left(p_{r, 0}(\varphi)\right)^{1-2^{-m-1}} .
\end{aligned}
$$

This completes the proof of Theorem 3.1. 
When $w_{1}(x)=w_{2}(x)$, the characterization of $\mathfrak{S}_{w_{1}, w_{2}}$ given by Theorem 3.1 reduces to the characterization of Beurling-Björck space $\mathfrak{S}_{w_{1}}$ given by Theorem 2.4. In particular, when $w_{1}(x)=w_{2}(x)=\ln (1+|x|)$, the characterization of $\mathfrak{S}_{w_{1}, w_{2}}$ reduces to the characterization of Schwartz space $\mathfrak{S}$.

Remark 3.2. The Fourier transform is a topological isomorphism between $\mathfrak{S}_{w_{1}, w_{2}}$ and $\mathfrak{S}_{w_{2}, w_{1}}$. As a consequence, the Fourier transform is also a topological isomorphism between the dual spaces $\mathfrak{S}_{w_{1}, w_{2}}^{\prime}$ and $\mathfrak{S}_{w_{2}, w_{1}}^{\prime}$.

Note that the dual spaces $\mathfrak{S}_{w_{1}, w_{2}}^{\prime}$ and $\mathfrak{S}_{w_{2}, w_{1}}^{\prime}$ are assigned to the weak topologies. For different pairs of admissible functions, the space $\mathfrak{S}_{w_{1}, w_{2}}$ has the following embedding properties.

Lemma 3.3. For every $w_{1}<w_{1}^{\prime}$ and $w_{2}<w_{2}^{\prime}$, one has

$$
\mathfrak{S}_{w_{1}^{\prime}, w_{2}^{\prime}} \hookrightarrow \mathfrak{S}_{w_{1}, w_{2}}
$$

Lemma 3.4. For $\alpha, \beta>1$, one has $\mathfrak{S}_{|x|^{1 / \alpha},|x|^{1 / \beta}} \subseteq S_{\alpha}^{\beta}$. As a consequence, $\left(S_{\alpha}^{\beta}\right)^{\prime} \subseteq \mathfrak{S}_{|x|^{1 / \alpha},|x|^{1 / \beta}}^{\prime}$.

\section{A representation theorem for functionals in the space $\mathfrak{S}_{w_{1}, w_{2}}^{\prime}$}

From Theorem 3.1, we can write

$$
\mathfrak{S}_{w_{1}, w_{2}}=\left\{\varphi: \mathbb{R}^{n} \longrightarrow \mathbb{C}: \varphi \text { is continuous and for all } k=0,1,2, \ldots, \mathcal{N}_{k}(\varphi)<\infty\right\},
$$

where $\mathcal{N}_{k}(\varphi)=\left\|e^{k w_{1}} \varphi\right\|_{\infty}+\left\|e^{k w_{2}} \widehat{\varphi}\right\|_{\infty}$.

Theorem 4.1. Given $L: \mathfrak{S}_{w_{1}, w_{2}} \rightarrow \mathbb{C}$, the following statements are equivalent:

(i) $L \in \mathfrak{S}_{w_{1}, w_{2}}^{\prime}$;

(ii) there exist two regular complex Borel measures $\mu_{1}$ and $\mu_{2}$ of finite total variation and $k \in$ $\{0,1,2, \ldots\}$ such that

$$
L=e^{k w_{1}} \mu_{1}+\mp\left[e^{k w_{2}} \mu_{2}\right]
$$

in the sense of $\mathfrak{S}_{w_{1}, w_{2}}^{\prime}$.

Proof. (i) $\Rightarrow$ (ii). Given $L \in \mathfrak{S}_{w_{1}, w_{2}}^{\prime}$, according to (4.1) there exist $k$ and $C$ so that

$$
L(\varphi) \leq C\left(\left\|e^{k w_{1}} \varphi\right\|_{\infty}+\left\|e^{k w_{2}} \widehat{\varphi}\right\|_{\infty}\right)
$$

for all $\varphi \in \mathfrak{S}_{w_{1}, w_{2}}$. Moreover, the map

$$
\begin{aligned}
\mathfrak{S}_{w_{1}, w_{2}} & \longrightarrow \mathcal{C}_{0} \times \mathcal{C}_{0}, \\
\varphi & \longrightarrow\left(e^{k w_{1}} \varphi, e^{k w_{2}} \widehat{\varphi}\right)
\end{aligned}
$$

is well defined, linear, continuous, and injective. Let $R$ be the range of this map, on which we define the map

$$
l_{1}(f, g)=L(\varphi)
$$


International Journal of Mathematics and Mathematical Sciences

where $f=e^{k w_{1}} \varphi, g=e^{k w_{2}} \widehat{\varphi}$ for a unique $\varphi \in \mathfrak{S}_{w_{1}, w_{2}}$. The map $l_{1}: \mathbb{R} \rightarrow \mathbb{C}$ is linear and continuous. By the Hahn-Banach theorem, there exists a functional $L_{1}$ in the topological dual $\left(\mathcal{C}_{0} \times \mathcal{C}_{0}\right)^{\prime}$ of $\mathcal{C}_{0} \times \mathcal{C}_{0}$ such that $\left\|L_{1}\right\|=\left\|l_{1}\right\|$ and the restriction of $L_{1}$ to $\mathcal{R}$ is $l_{1}$.

Since the spaces $\left(\mathcal{C}_{0} \times \mathcal{C}_{0}\right)^{\prime}$ and $\mathcal{C}_{0}^{\prime} \times \mathcal{C}_{0}^{\prime}$ are isomorphic as Banach spaces, we can write $L_{1}(f, g)=L_{1}(f, 0)+L_{1}(0, g)$. Using Theorem 2.6, there exist regular complex Borel measures $\mu_{1}$ and $\mu_{2}$ of finite total variation such that

$$
L_{1}(f, g)=\int_{\mathbb{R}^{n}} f d \mu_{1}+\int_{\mathbb{R}^{n}} g d \mu_{2}
$$

for all $(f, g) \in \mathcal{C}_{0} \times \mathcal{C}_{0}$. If $(f, g) \in \mathcal{R}$, then we conclude that

$$
L(\varphi)=\int_{\mathbb{R}^{n}} e^{k w_{1}} \varphi d \mu_{1}+\int_{\mathbb{R}^{n}} e^{k w_{2}} \widehat{\varphi} d \mu_{2}
$$

for all $\varphi \in \mathfrak{S}_{w_{1}, w_{2}}$. In the sense of $\mathfrak{S}_{w_{1}, w_{2}}^{\prime}$

$$
L=e^{k w_{1}} \mu_{1}+\mp\left[e^{k w_{2}} \mu_{2}\right] .
$$

(ii) $\Rightarrow$ (i). If $\mu_{1}$ and $\mu_{2}$ are two regular complex Borel measures satisfying (ii) and $\varphi \in \mathfrak{S}_{w_{1}, w_{2}}$, then

$$
L(\varphi)=\int_{\mathbb{R}^{n}} e^{k w_{1}} \varphi d \mu_{1}+\int_{\mathbb{R}^{n}} e^{k w_{2}} \widehat{\varphi} d \mu_{2}
$$

This implies that

$$
\begin{aligned}
|L(\varphi)| & \leq\left|\int_{\mathbb{R}^{n}} e^{k w_{1}} \varphi d \mu_{1}\right|+\left|\int_{\mathbb{R}^{n}} e^{k w_{2}} \hat{\varphi} d \mu_{2}\right| \\
& \leq\left|\mu_{1}\right|\left(\mathbb{R}^{n}\right)\left\|e^{k w_{1}} \varphi\right\|_{\infty}+\left|\mu_{2}\right|\left(\mathbb{R}^{n}\right)\left\|e^{k w_{2}} \hat{\varphi}\right\|_{\infty} \\
& \leq C\left(\left\|e^{k w_{1}} \varphi\right\|_{\infty}+\left\|e^{k w_{2}} \widehat{\varphi}\right\|_{\infty}\right) .
\end{aligned}
$$

It may be noted that $\mu_{1}$ and $\mu_{2}$, employed to obtain the above inequality, are of finite total variations. This completes the proof of Theorem 4.1.

Remark 4.2. When $w_{1}(x)=w_{2}(x)=(1+|x|)^{k}$, (4.2) becomes

$$
L=(1+|x|)^{k} \mu_{1}+\mathcal{F}\left[(1+|\xi|)^{k} \mu_{2}\right] \text {, }
$$

which gives a representation for the tempered distributions.

As consequence of Lemma 3.4, we can view the functionals in $\left(S_{a}^{b}\right)^{\prime}$ as functionals in the space $\mathfrak{S}_{w_{1}, w_{2}}^{\prime}$. Then as a result we can characterize $\left(S_{\alpha}^{\beta}\right)^{\prime}$ using Theorem 4.1.

Corollary 4.3. Let $\alpha, \beta>1$. Then any $L \in\left(S_{\alpha}^{\beta}\right)^{\prime}$ can be written as

$$
L=e^{k|x|^{1 / \alpha}} \mu_{1}+\mathcal{F}\left[e^{k|\xi|^{1 / \beta}} \mu_{2}\right]
$$

which characterizes the dual space $\left(S_{\alpha}^{\beta}\right)^{\prime}$. 


\section{References}

[1] A. Beurling, Quasi-Analyticity and General Distributions, Lectures 4 and 5, Multigraphed Lecture Notes, American Mathematical Society Summer Institute, Stanford, Calif, USA, 1961.

[2] G. Björck, "Linear partial differential operators and generalized distributions," Arkiv för Matematik, vol. 6, pp. 351-407, 1966.

[3] L. Hörmander, Linear Partial Differential Operators, vol. 116 of Die Grundlehren der mathematischen Wissenschaften, Springer, Berlin, Germany, 1963.

[4] M. Andersson and B. Berndtsson, "Almost holomorphic extensions of ultradifferentiable functions," Journal d'Analyse Mathématique, vol. 89, pp. 337-365, 2003.

[5] J. Alvarez and H. M. Obiedat, "Characterizations of the Schwartz space $\mathfrak{S}$ and the Beurling-Björck space $\mathfrak{S}_{W}, "$ "Cubo, vol. 6, no. 4, pp. 167-183, 2004.

[6] S.-Y. Chung, D. Kim, and S. Lee, "Characterization for Beurling-Björck space and Schwartz space," Proceedings of the American Mathematical Society, vol. 125, no. 11, pp. 3229-3234, 1997.

[7] W. Rudin, Functional Analysis, International Series in Pure and Applied Mathematics, McGraw-Hill, New York, NY, USA, 2nd edition, 1991.

[8] J. J. Duistermaat, Fourier Integral Operators, Courant Institute of Mathematical Sciences, New York, NY, USA, 1973.

[9] J. Hadamard, "Sur le module maximum d'une function et de ses dérivés," Comptes Rendus de l'Académie des Sciences, vol. 41, pp. 68-72, 1914.

[10] E. Landau, "Einige ungleichungen für zweimal diffentiierbare Funktionen," Proceedings of the London Mathematical Society, vol. 13, no. 1, pp. 43-49, 1914. 


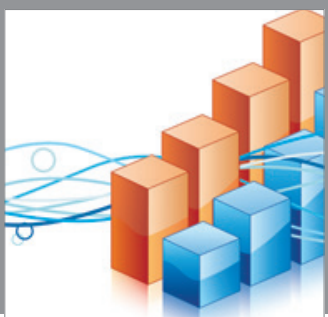

Advances in

Operations Research

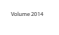

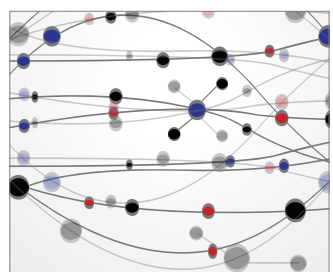

\section{The Scientific} World Journal
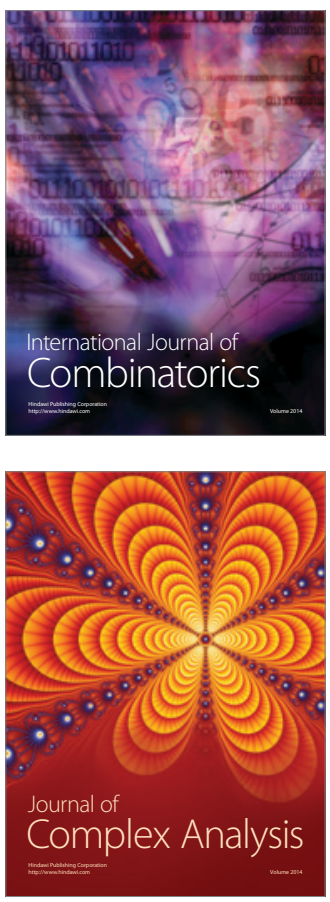

International Journal of

Mathematics and

Mathematical

Sciences
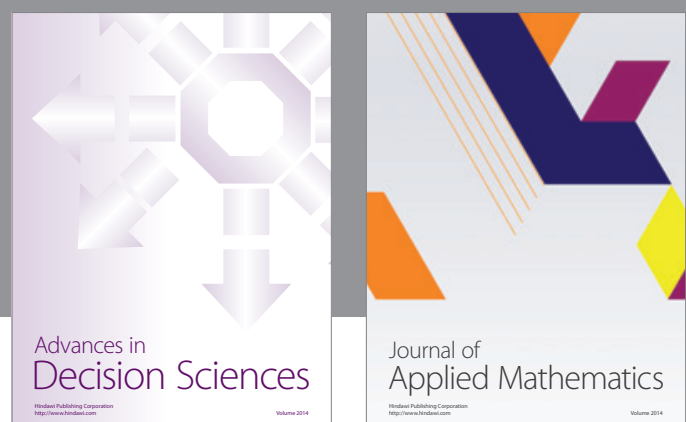

Journal of

Applied Mathematics
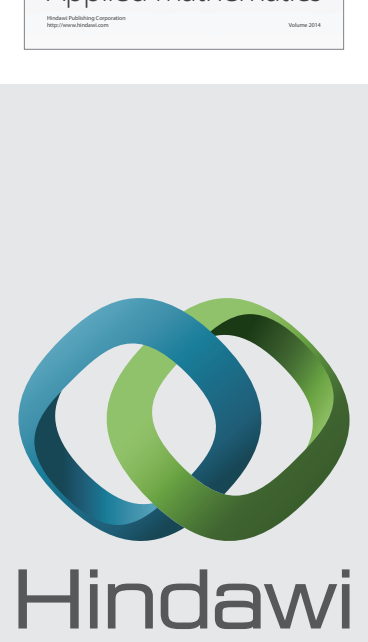

Submit your manuscripts at http://www.hindawi.com
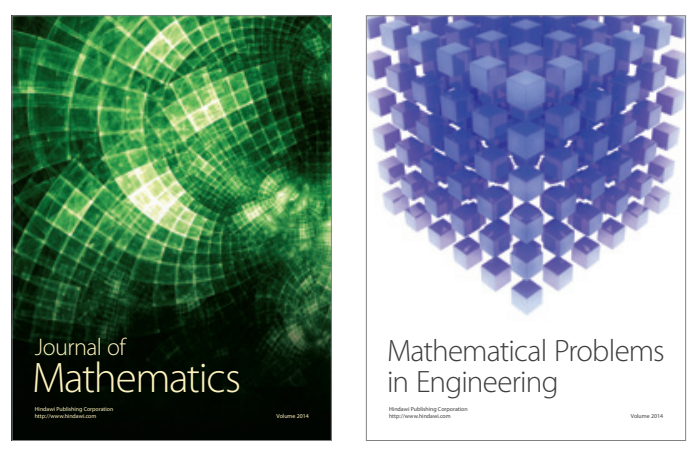

Mathematical Problems in Engineering
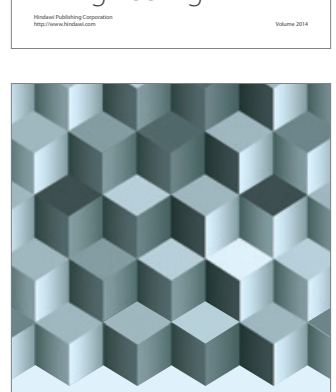

Journal of

Function Spaces
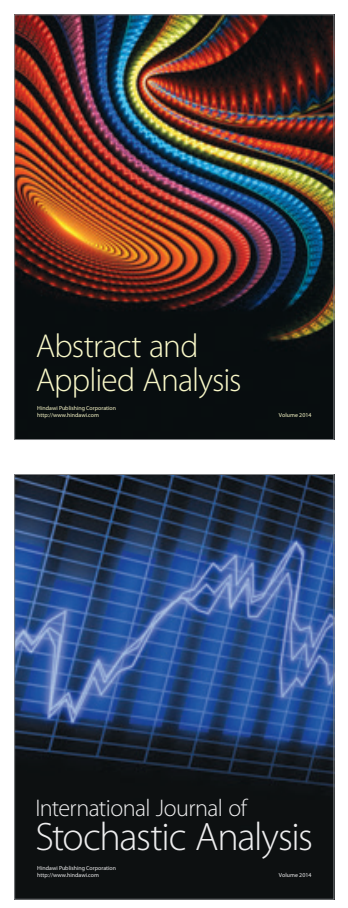

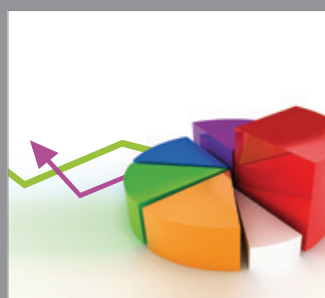

ournal of

Probability and Statistics

Promensencen
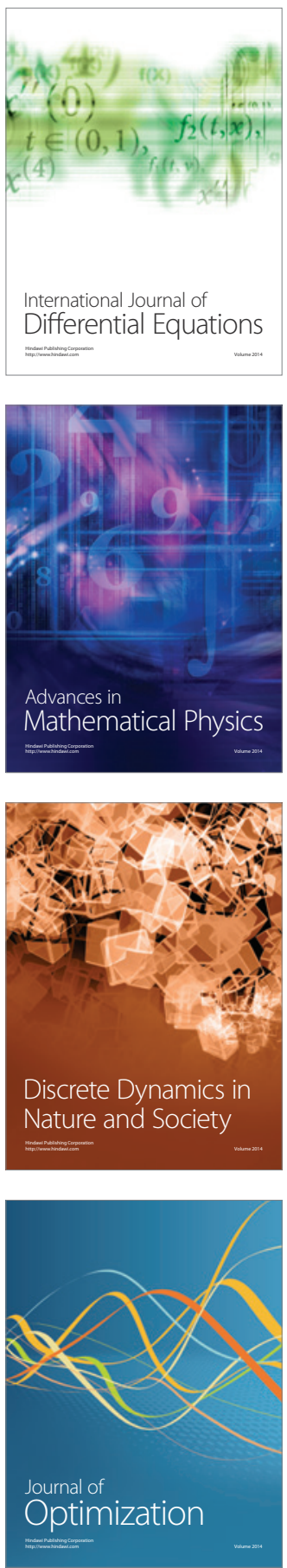